\title{
Évaluation du potentiel prolifératif de six cultivars de bananier (cv. AAB, ABB, et AAA) par macropropagation en République Démocratique du Congo.
}

\author{
Bangata Bitha nyi Mbunzu ${ }^{1 *}$, Mobambo Kitume Ngongo¹, Kasongo Munyinga1, Shungu Dambo², Vuvu \\ Kubangisa2, Vangu Phaka², Omondi Aman ${ }^{3}$, Staver Charles ${ }^{4}$ \\ ${ }_{1}^{1}$ Département de Phytotechnie, Faculté des Sciences Agronomiques, Université de Kinshasa, BP.117 Kinshasa XI, \\ République Démocratique du Congo. \\ ${ }^{2}$ Institut National pour l'Étude et la Recherche Agronomiques (INERA), République Démocratique du Congo. \\ ${ }^{3}$ Bioversity International, Burundi. \\ ${ }^{4}$ Bioversity International, France. \\ *Auteur correspondant : Email : jeanchristian.bangata@unikin.ac.cd
}

Original submitted in on $6^{\text {th }}$ April 2018. Published online at www.m.elewa.org on $31^{\text {st }}$ July 2018 https://dx.doi.org/10.4314/jab.v127i1.3

\section{RESUME}

Objectifs: L'objectif était celui d'améliorer la technique de macropropagation pour la production du matériel végétal de plantation de bananiers, en comparant les différents cultivars en vue d'identifier le(s) cultivar(s) qui répond(ent) mieux à cette méthode.

Méthodologie et résultats : Le test ELISA a été utilisé en vue de cribler les échantillons des plantes, en rapport avec la maladie de Bunchy Top (BBTD). Suivant le dispositif complètement randomisé avec trois répétitions dans les propagateurs, les résultats obtenus ont montré que le nombre total de plantules sevrées par explants le plus élevé a été observé chez les bananiers à cuire Saba et Cardaba respectivement avec 35 et 34 plantules, suivi du plantain Bubi avec 31 plantules. Tandis que, le nombre total de plantules le plus faible a été observé chez le Dessert Gros Michel avec 23 plantules.

Conclusion et application des résultats: Au regard des résultats obtenus, tous ces cultivars peuvent être retenus et utilisés dans la technique de macro-propagation en vue d'augmenter la production de matériel de plantation de la culture de bananier en un temps record mais en mettant un accent particulier sur le plantain BUBI pour la ville de Kinshasa et ses environs. Ainsi, le fait que ces matériels biologiques testés soient indemnes de toute contamination virale (BBTV), ils constituent donc le matériel de choix en vue de leur macropropagation, étape préliminaire nécessaire à la mise en place d'un champ expérimental du système agroforestier.

Mots clés : Méthode PIF, prolifération, cultivars, bananiers, RDC. 


\section{Assessment of the prolific potential of six cultivars of banana tree (cv. $A A B, A B B$, and $A A A$ ) by macropropagation in Democratic Republic of Congo. ABSTRACT}

Objectives: The objective was to improve the technique of macropropagation for the production of the plant material of plantation of banana trees by comparing the different cultivars in order to identify the cultivar(s) that respond better to this method.

Methodology and results: The ELISA test has been used in order to sift the samples of the plants, in relation with the disease of Bunchy Top (BBTD). According to the completely randomized device with three repetitions in the propagators, the findings showed that the total number of plantules weaned by most elevated explants has been observed in the banana trees to cook Saba and Cardaba respectively with 35 and 34 plantules, followed from the Bubi plantain with 31 plantules. However, the total number of the lowest plantules has been observed at the Dessert Gros Michel with 23 plantules.

Conclusion and application of the results: With regard to the findings, all these cultivars can be kept and can be used in the technique of macro - propagation in order to increase the production of the material of the plantation of the banana tree in due time by putting a particular emphasis on the BUBI plantain for Kinshasa city and its surroundings. Thus, the fact that these tested biological materials are unscathed of all viral contamination (BBTV), they constitute the choice material for the sake of their macropropagation, preliminary and necessary stage to the implementation up of an experimental field of the agroforestry system.

Keywords : Method PIF, proliferation, cultivars, banana trees, DRC.

\section{INTRODUCTION}

Dans le monde, la banane dessert et le plantain jouent un rôle essentiel dans la sécurité alimentaire de plus de 400 millions de personnes dans les pays en développement des zones tropicales et contribuent de manière significative à la sécurité alimentaire et à la diversification des revenus dans les zones rurales (Bakelana et Muyunga, 1998; Temple et al., 2002). Ainsi, ils contribuent, de façon significative à la lutte contre la pauvreté (Nkendah et Akeyeampong, 2003, Arias et al., 2003). En République Démocratique du Congo (RDC), le plantain constitue actuellement, le troisième produit vivrier après le manioc et le maïs, tandis que les bananes douces représentent les fruits les plus importants du pays, soit $1 / 3$ de la production fruitière totale (Mobambo et al., 2010 ; Dhed'adjailo et al., 2011). Cependant, les plantations de bananier plantain sont de faible superficie à cause de l'indisponibilité du matériel végétal de bonne qualité. Ceci ne permet pas de rentabiliser l'effort fourni par les paysans et découragent ces derniers (NgoSamnick, 2011). En effet, la production ainsi que l'approvisionnement en plantules saines des bananiers est un problème sérieux en milieu périurbains et ruraux (Kasyoka et al., 2010). Le rejetonnage comme mode de propagation présente des inconvénients notamment la lenteur dans la multiplication du fait que le nombre de rejet par plante est limité (dépasse rarement 5 et même 3 pour le plantain), l'hétérogénéité du matériel de propagation et enfin la lourdeur et le volume des rejets faisant que leur transport soit coûteux (Kwa, 2003). A cet effet, plusieurs techniques existent pour augmenter la production de rejets sur un pied de bananier, les plus simples sont pratiquées au champ, mais leur rendement reste faible (Sadom et al., 2010). Par ailleurs, la solution de vitro plants qui consiste à disposer et utiliser du matériel amélioré en quantité suffisante, indemne de maladies, n'est pas à la portée des agriculteurs traditionnels (Youmbi et Ngaha, 2004). Devant de telles situations, la majorité de producteurs se tournent vers la nature à la recherche de solution pouvant répondre à leurs besoins (Manzur-marcia, 2001). Or il existe aujourd'hui des techniques éprouvées disponibles et accessibles à tous (Njeri Njau et al. 2012). La technique des Plants Issus des Fragments de tiges (PIF) conduit à une production rapide d'un matériel de type vivo-plant en seulement trois ou quatre mois à n'importe quel moment de l'année sans difficulté (Bonte et al., 1995 ; Kwa, 1998b et 2007). II existe plusieurs cultivars des bananiers et chacun d'eux a son mode de vie et son 
cycle de rejetonnage particulier. Malgré son importance pour le développement, très peu d'études ont été effectuées en RDC sur la production de matériel de plantation en vue de l'expansion des espaces cultivables et de l'organisation de la filière. La présente étude se focalise sur l'amélioration de la technique de macro-propagation en comparant la performance de six différents cultivars de bananier

\section{MATERIEL ET METHODES}

Site expérimental : Cette étude a été menée au Jardin Expérimental du Département de Phytotechnie de la Faculté des Sciences Agronomiques (Université de Kinshasa), au plateau du Mont-Amba (coordonnées géographiques : $04^{\circ} 25^{\prime} 06,6^{\prime \prime}$ de latitude Sud, $018^{\circ} 18^{\prime}$ 24,8 " de longitude Est et $433 \mathrm{~m}$ d'altitude) en RDC.
(Musa sp, cv. AAB ; ABB et AAA) dans la prolifération de plantules en vue d'identifier le meilleur cultivar qui répond mieux à la méthode PIF et pouvant permettre aux agriculteurs d'accroître leur production. La technique des PIF permet d'exploiter la plupart des bourgeons de la plante, que ceux-ci soient visibles ou non (Meutchieye, 2009).

L'expérience a été réalisée dans deux propagateurs bien aménagés et construits en blocs de ciment dont les longueurs étaient respectivement de $12 \mathrm{~m}$ et $9,6 \mathrm{~m}$ et de $1,20 \mathrm{~m}$ de largeur, divisés respectivement en 6 et 3 compartiments égaux (figure 1).
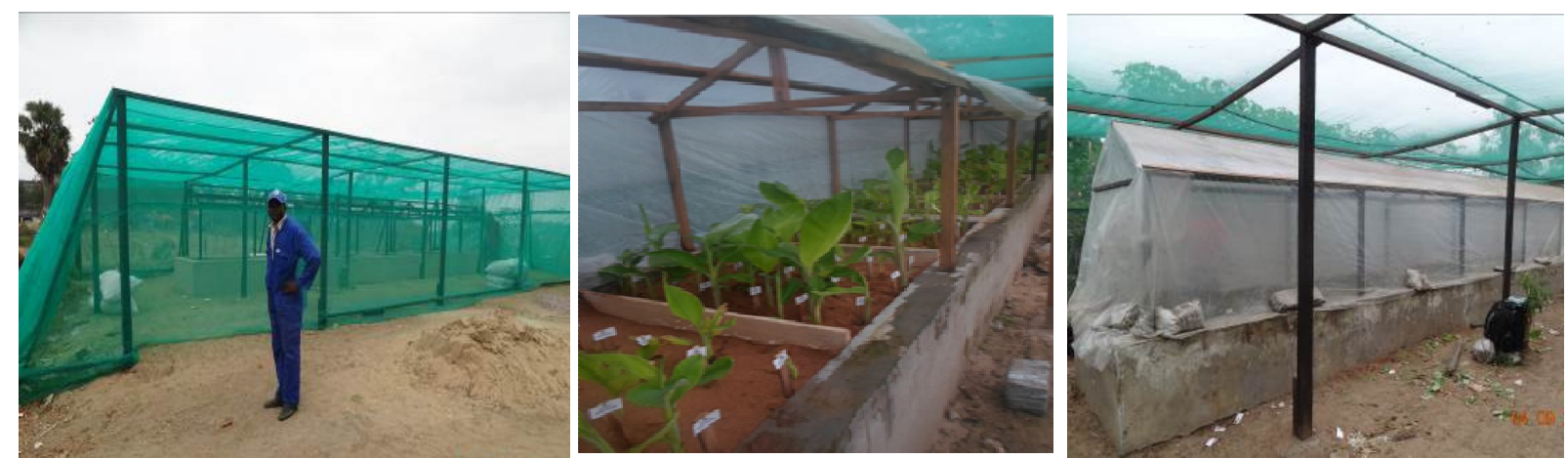

Figure 1. Construction de propagateurs et de l'ombrière

La hauteur des bacs était de $0,6 \mathrm{~m}$. Le fond des bacs est isolé du sol pour éviter tout contact direct entre le substrat et la terre. Nous avons placé une couche de béton au fond des bacs avec des tuyauteries d'évacuation d'eau d'arrosage. L'expérimentation a été réalisée au cours de la période allant du 01 juin au 23 octobre 2015. La température pendant la période de l'essai a varié entre 28,6 et $45,9^{\circ} \mathrm{C}$ présentant une moyenne de $36,2^{\circ} \mathrm{C}$. Quant à l'humidité relative, elle a varié entre $40,8 \%$ et $100,0^{\circ} \mathrm{C}$ présentant une moyenne de $85,9^{\circ} \mathrm{C}$.

Matériel végétal : Six cultivars de bananier et plantain ont été utilisés à savoir pour le plantain (AAB): Bubi et Ndongila ; pour le bananier à table (AAA) : Gros Michel et Yangambi km5; enfin pour le bananier à cuire (ABB) : Saba et Cardaba. Le choix a été porté sur ces cultivars à cause de leur appréciation par les paysans cultivateurs du Kongo-Central et par les commerçants acheteurs. Ce matériel au préalable testé (test d'ELISA) nous a été fourni par le projet Bioversity International en provenance de I'INERA-Mvuazi dans la province du Kongo-Central.

Méthodes : L'étude a été menée suivant un dispositif en blocs complètement randomisés comportant trois répétitions. Chaque répétition comprenait six compartiments représentant six cultivars. Chaque répétition comportait 180 explants soit un total de 540 boutures pour les 18 compartiments. Les explants ont été installés aux écartements de $10 \mathrm{~cm} \times 5 \mathrm{~cm}$. Les bacs ont été couverts hermétiquement des plastiques résistants et transparents. Les propagateurs étaient abrités par une ombrière qui permet de réduire l'impact des rayons incidents du soleil sur les plants. Le test d'ELISA a été utilisé en vue de cribler les échantillons des plants de bananier en rapport avec la maladie de Bunchy Top (BBTD). Pour prélever les rejets, nous avons bien fait la fouille autour du plant ciblé (testé). 

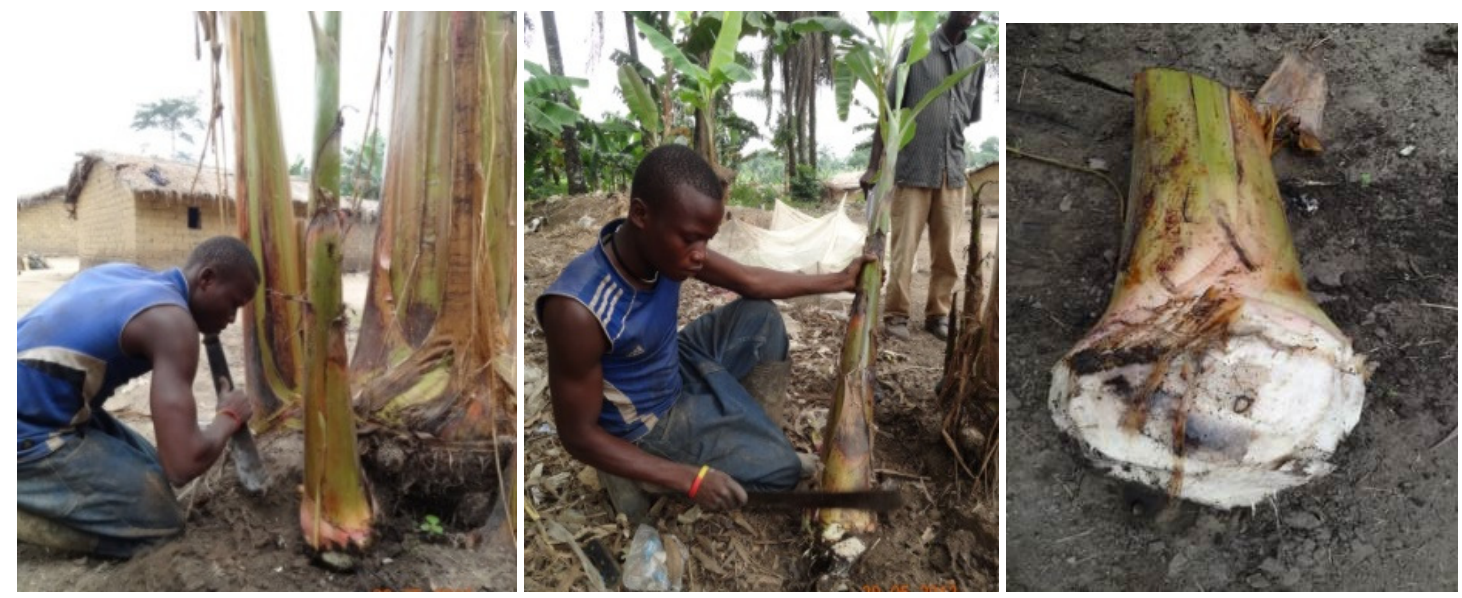

Figure 2. Extraction et toilettage de rejets

Parage à blanc : nous avons paré les rejets à l'aide d'un couteau de cuisine jusqu'à obtenir la couleur blanche. Le but de cette opération était de débarrasser du bulbe toutes les racines y compris les galeries de charançons.
Décorticage : les gaines foliaires ont été enlevées l'une après l'autre tout en pratiquant le découpage. Celui-ci n'était pas très haut de peur qu'il freine l'œil de pouvoir émerger, non plus être très bas car il risque d'endommager l'œil.
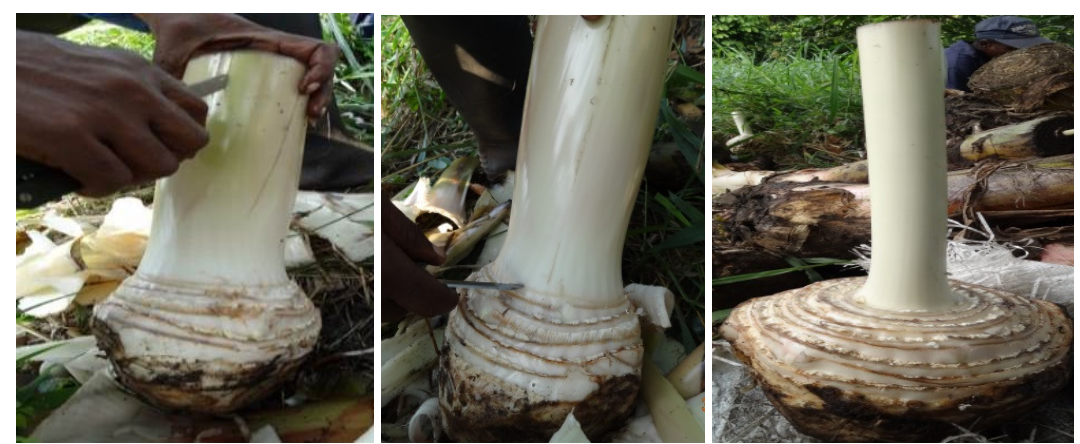

Figure 3. Décorticage d'un rejet en explant

Après ces opérations, nous avons préparé une solution désinfectante $[40 \mathrm{~g}$ de fongicide (Fongicalme) $+50 \mathrm{ml}$ d'insecticide (Verso 480)] dans 10 litres d'eau, dans laquelle les explants ont été trempés pendant environ 3 à 5 minutes. Enlevés de la solution, les explants sont étalés sur un film plastique pendant $48 \mathrm{~h}$ pour l'égouttage.

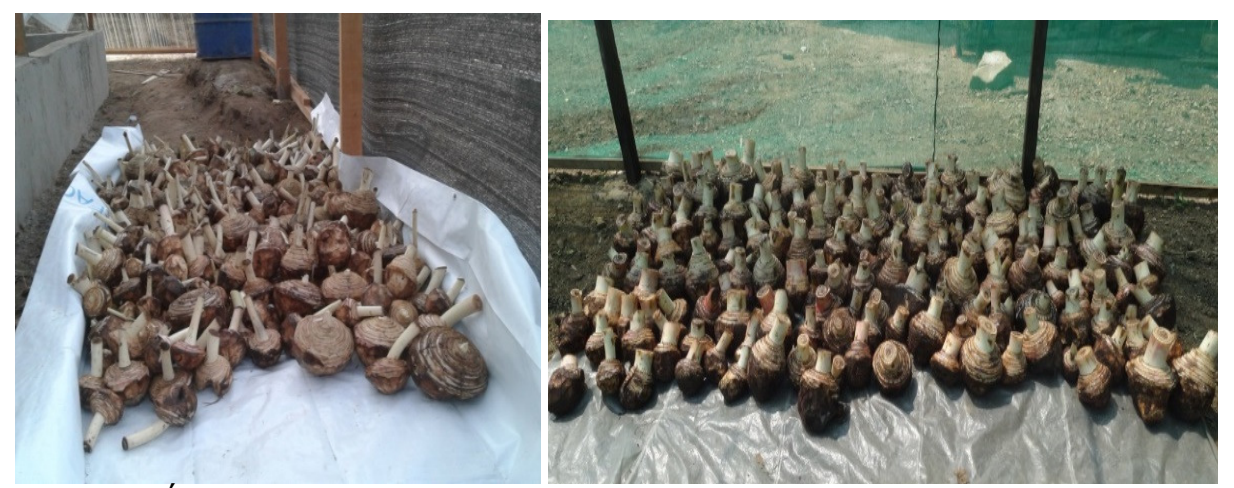

Figure 4 : Égouttage des explants pendant 48 heures sous ombrières 
Rajeunissement et application des incisions: le rajeunissement a consisté à la suppression de bout de pseudo-tige laissé après le décorticage. Il s'en est suivi
2 à 4 incisions droites en croix au centre de l'explant tout en repérant la partie centrale de l'explant de rejet lors de rajeunissement par des coupes.
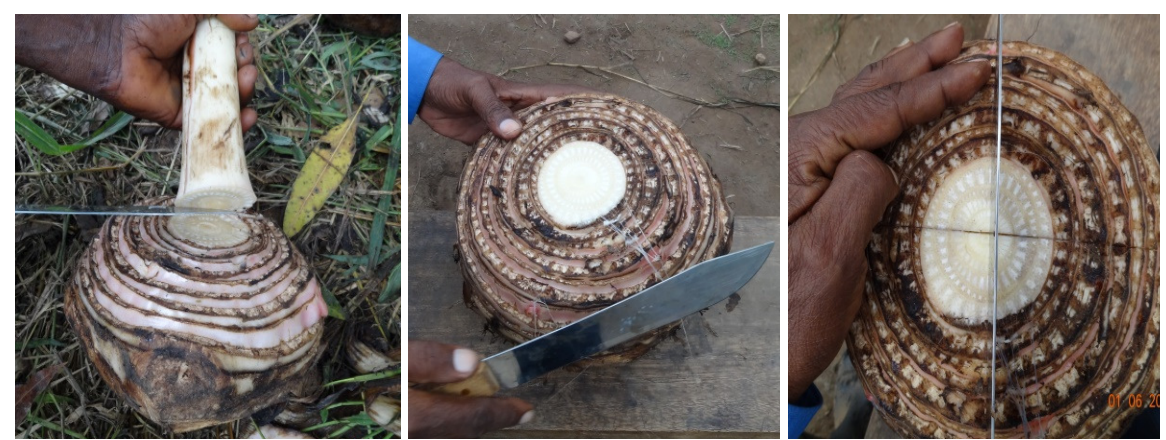

Figure 5 : Rajeunissement et pose des incisions pour détruire le méristème apical

Mise en bac : Les explants ayant subi le rajeunissement et application des incisions ont été installés dans les six compartiments du bac de germination comprenant chacun la sciure de bois comme substrat d'enracinement. Ils ont été installés aux écartements de $10 \mathrm{~cm} \times 5 \mathrm{~cm}$ et recouverts d'une épaisseur de 2 à $5 \mathrm{~cm}$ de la sciure audessus de la surface des explants. Aucune hormone n'avaient été ajoutée à la sciure de bois, ni avant, ni après l'ensemencement. Enfin, le bac a été hermétiquement fermé avec un papier plastique transparent, ceci dans le but de créer un microclimat (augmenter la température) favorable à la propagation. Le bac de germination a été préalablement arrosé suffisamment pendant deux jours au moins. La fréquence d'arrosage a été de deux fois par semaine.

Sevrage des Plants : 35 jours après la mise en germoir, nous avons procédé au sevrage c'est- à- dire détacher les plants de l'explant à l'aide d'un couteau très fin. Puis les repiquer dans des sachets en polyéthylène d'une contenance d'environ $1 \mathrm{Kg}$ de terreau stérilisé, pour assurer le développement des jeunes plants de bananier.
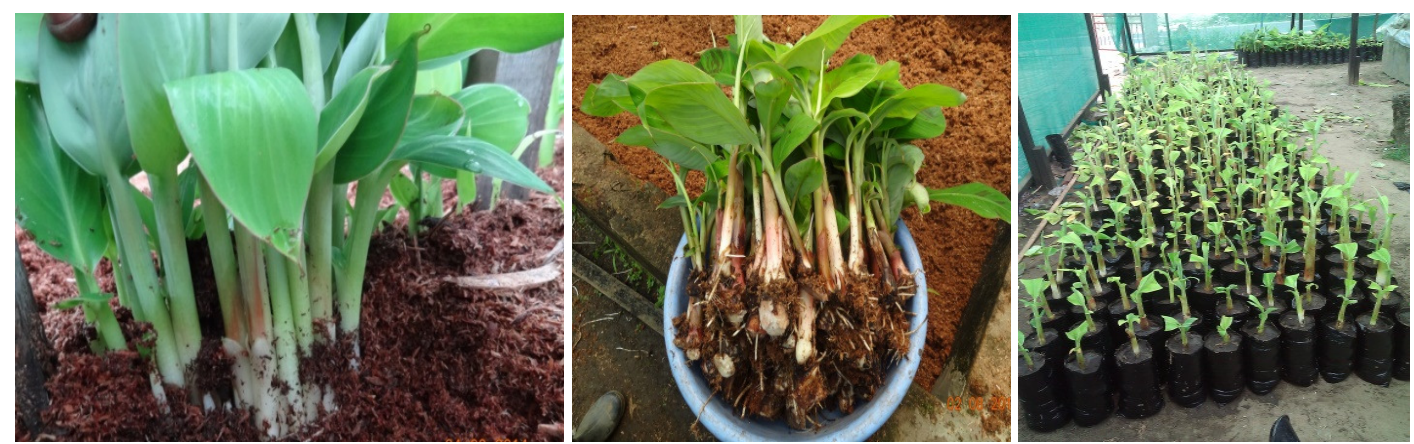

Figure 6 : Plantules, sevrées, repiquées en sachets et classées sous ombrière

Au cours de cette étude, la température et l'humidité relative ont été mesurées dans le germoir. Ces données portant sur l'enregistrement des températures maximales, optimales et minimales et de l'humidité relative de l'enceinte du germoir ont été comparées à celles ambiantes observées par le poste d'observations météorologiques du site de l'étude. 5 appareils tinytags nous ont servi pour cette fin.
Analyse des résultats : Pour chaque cultivar étudié, les données collectées ont été analysées selon la méthode de l'analyse de variance, ANOVA au seuil de probabilité de $5 \%$. Le test de la plus petite différence significative (PPDS) était utilisé pour comparer les résultats des différents cultivars utilisés. Tous ces tests sont effectués à l'aide des logiciels Excel (version 2010) et Statistix (version 8.0). 


\section{RESULTATS}

Tableau 1. Les résultats relatifs aux paramètres végétatifs observés au cours de l'expérimentation sur la détermination du taux de multiplication de plantules formées par les explants de bananiers en fonction de cultivars à partir d'un seul substrat (sciure de bois).

\begin{tabular}{l|c|c|c|c|c}
\hline Traitements & $\begin{array}{c}\text { HPS } \\
(\mathrm{cm})\end{array}$ & $\begin{array}{c}\text { DCPS } \\
(\mathrm{cm})\end{array}$ & NFFS & NRFS & LRFS (cm) \\
\hline Plantain (Ndongila) & $22,6 \pm 1,5 \mathrm{a}$ & $2,9 \pm 0,1 \mathrm{abc}$ & $3,1 \pm 0,3 \mathrm{a}$ & $2,6 \pm 0,3 \mathrm{bc}$ & $3,9 \pm 0,8 \mathrm{bc}$ \\
Plantain (Bubi) & $19,2 \pm 1,5 \mathrm{ab}$ & $2,8 \pm 0,2 \mathrm{bc}$ & $2,3 \pm 0,1 \mathrm{~b}$ & $3,1 \pm 0,7 \mathrm{abc}$ & $7,3 \pm 1,9 \mathrm{a}$ \\
Banane à cuire (Saba) & $17,1 \pm 1,9 \mathrm{~b}$ & $2,3 \pm 0,4 \mathrm{c}$ & $3,1 \pm 0,4 \mathrm{a}$ & $3,9 \pm 0,7 \mathrm{ab}$ & $2,5 \pm 0,5 \mathrm{c}$ \\
Banane à cuire (Cardaba) & $23,0 \pm 0,8 \mathrm{a}$ & $3,3 \pm 0,3 \mathrm{ab}$ & $2,2 \pm 0,1 \mathrm{~b}$ & $4,1 \pm 1,1 \mathrm{a}$ & $8,1 \pm 1,6 \mathrm{a}$ \\
Dessert (Gros Michel) & $22,7 \pm 1,2 \mathrm{a}$ & $3,1 \pm 0,2 \mathrm{ab}$ & $3,1 \pm 0,3 \mathrm{a}$ & $3,3 \pm 0,7 \mathrm{abc}$ & $4,6 \pm 1 \mathrm{bc}$ \\
Dessert (Yangambi km5) & $21,6 \pm 1,9 \mathrm{ab}$ & $3,6 \pm 0,8 \mathrm{a}$ & $2,5 \pm 0,5 \mathrm{~b}$ & $2,4 \pm 0,6 \mathrm{c}$ & $5,1 \pm 0,1 \mathrm{~b}$ \\
CV (\%) & 11,08 & $\mathbf{8 , 2 4}$ & $\mathbf{4 , 5 6}$ & $\mathbf{6 , 9 4}$ & $\mathbf{3 , 1 8}$ \\
\hline
\end{tabular}

Légende : HPS = Hauteur des plantules lors du sevrage $;$ DCPS = Diamètre au collet des plantules $;$ NFFS $=$ Nombre de feuilles formées ; NRFS = Nombre de racines formées ; LRFS = Longueur de racines formées. Les chiffres dans les colonnes suivis de mêmes lettres ne sont pas significativement différents selon le test de la Plus Petite Différence Significative (PPDS) à $5 \%$ de probabilité.

Les résultats ont montré que les valeurs observées sur la hauteur de tous les cultivars utilisés sont similaires sauf chez le cultivar $\mathrm{ABB} / \mathrm{Saba}$ qui a présenté une hauteur inferieure $(17,11 \mathrm{~cm})$. II en est de même concernant le diamètre au collet. Cependant, nous remarquons de ces résultats qu'il existe une relation étroite entre le diamètre au collet et la hauteur des plantules formées, plus la hauteur des plantules formées est grande, plus sera leur diamètre au collet. Par rapport au nombre de feuilles, il ressort du tableau 1 qu'il existe de différence significative au seuil de probabilité de $5 \%$, entre les six cultivars mis en essai. En ce qui concerne le nombre de racines formées, le nombre le plus élevé a été constaté chez deux cultivars $\mathrm{ABB} / \mathrm{à} \mathrm{cuire} \mathrm{(Cardaba}=4$ et Saba $=3,85$ ) et le nombre de racines le plus faible a été constaté chez Musa sp, cv. AAA/Dessert (Yangambi km5 = 2). Quant à la longueur de racines formées, les valeurs les plus élevées ont été observées chez Musa sp, cv. ABB/à cuire (Cardaba avec $8,1 \mathrm{~cm}$ ). Par contre, Musa sp, cv. ABB/à cuire (Saba) a présenté les racines ayant la longueur la plus faible avec $2,49 \mathrm{~cm}$.

Tableau 2. Les résultats relatifs aux paramètres de prolifération observés au cours de l'expérimentation sur la détermination du taux de multiplication de plantules formées en fonction de cultivar à partir d'un seul substrat (sciure de bois).

\begin{tabular}{l|l|l|l|l|l}
\hline Traitements & $\begin{array}{l}\text { DR } \\
\text { (Jours) }\end{array}$ & $\begin{array}{l}\text { TR } \\
(\%)\end{array}$ & NPS-1mois & NTPOE & NTPOC/m $\mathbf{m}^{2}$ \\
\hline Plantain (Ndongila) & $21,7 \pm 0,6 \mathrm{~b}$ & $87,8 \pm 1,5 \mathrm{a}$ & $5,1 \pm 0,7 \mathrm{ab}$ & $29,8 \pm 1,9 \mathrm{c}$ & $575,2 \pm 14 \mathrm{bc}$ \\
Plantain (Bubi) & $22,0 \pm 1 \mathrm{~b}$ & $89,1 \pm 1,7 \mathrm{a}$ & $6,0 \pm 1,4 \mathrm{a}$ & $30,7 \pm 1,6 \mathrm{bc}$ & $594,9 \pm 18,7 \mathrm{~b}$ \\
Banane à cuire (Saba) & $20,0 \pm 1 \mathrm{c}$ & $92,2 \pm 1,8 \mathrm{a}$ & $7,0 \pm 1,8 \mathrm{a}$ & $35,5 \pm 1,8 \mathrm{a}$ & $673,0 \pm 21,4 \mathrm{a}$ \\
Banane à cuire (Cardaba) & $19,7 \pm 1,5 \mathrm{c}$ & $90,5 \pm 1,2 \mathrm{a}$ & $6,2 \pm 1,4 \mathrm{a}$ & $33,7 \pm 1,1 \mathrm{ab}$ & $603,1 \pm 11,2 \mathrm{~b}$ \\
Dessert (Gros Michel) & $21,7 \pm 0,6 \mathrm{~b}$ & $89,3 \pm 1 \mathrm{a}$ & $\mathbf{1 , 8 \pm 0 , 4 \mathrm { c }}$ & $22,6 \pm 1,7 \mathrm{~d}$ & $384,3 \pm 18,1 \mathrm{~d}$ \\
Dessert (Yangambi km5) & $24,3 \pm 1,5 \mathrm{a}$ & $86,7 \pm 1,2 \mathrm{a}$ & $3,5 \pm 0,3 \mathrm{bc}$ & $28,8 \pm 1,2 \mathrm{c}$ & $521,1 \pm 17,7 \mathrm{c}$ \\
CV (\%) & $\mathbf{8 , 1 5}$ & $\mathbf{1 3 , 0 1}$ & $\mathbf{3 , 7 5}$ & $\mathbf{9 , 3 2}$ & $\mathbf{6 , 2 2}$ \\
\hline
\end{tabular}

Légende : $D R=$ Durée de reprise $; T R=$ Taux de reprise; NPS-1mois = Nombre de Plantules Sevrées après1 mois d'incubation ; NTPOE = Nombre total des plantules obtenues par explant et NTPOC $/ \mathrm{m}^{2}=$ Nombre total de plantules obtenues par cultivar $/ \mathrm{m}^{2}$. Les chiffres dans les colonnes suivis de mêmes lettres ne sont pas significativement différents selon le test de la Plus Petite Différence Significative (PPDS) à $5 \%$ de probabilité.

Le résultat relatif à la durée de reprise de six cultivars indique que tous les cultivars utilisés sont de bonne qualité car leur durée de reprise est autour de 3 semaines. Cependant, l'analyse statistique au seuil de probabilité $5 \%$ révèle des différences significatives entre les cultivars (PPDS $=0,92$ ). La durée de reprise la plus courte a été enregistrée chez les explants issus de bananiers à cuire Saba et Cardaba (20 jours). En ce qui concerne le taux de 

ABB, et AAA) par macropropagation en République Démocratique du Congo.

reprise, les résultats issus de l'analyse statistique au seuil de probabilité $5 \%$ ne révèle aucune différence significative entre les cultivars (PPDS $=13,29)$. En outre, ces résultats indiquent que tous les cultivars utilisés sont de bonne qualité car leur taux de reprise est supérieur à $85 \%$. Par rapport au nombre des plantules sevrées après un mois d'incubation, il ressort du tableau 2 que le nombre de plantules sevrées par fragment le plus élevé a été observée chez les explants issus de deux bananiers à cuire (cv. ABB) et du plantain Bubi (cv. AAB) respectivement avec 7 et 6 plantules. Par contre, le nombre des plantules sevrées le plus faible a été constaté chez les explants issus de bananier Dessert Gros Michel (cv. AAA) avec 2 plantules. L'analyse statistique au seuil de probabilité $5 \%$ révèle de différences significatives entre

\section{DISCUSSION}

Les résultats obtenus sur le nombre de plantules ont montré que tous les cultivars de bananier ont le potentiel de prolifération des plantules en quantité en conditions semi-contrôlées (propagateurs). Cependant, cette prolifération est fortement dépendante du cultivar. Par rapport aux six cultivars testés, le Gros Michel a montré des résultats médiocres avec une faible productivité. En revanche, les explants de Saba se sont démarqués des autres en donnant le nombre des plantules supérieur par rapport à ceux d'autres cultivars soumis à l'étude. Cette différence peut être due à l'effet (dominance) de double génome $B$ qui fait que la prolifération soit importante et aux caractères intrinsèques de chaque cultivar. L'étude de la réponse variétale de cultivars de bananiers à la technique des PIF a montré que tous les cultivars ne réagissaient pas de la même façon. Ainsi les bananiers à cuire Saba et Cardaba seraient plus aptes à proliférer in vivo que tous les cultivars testés, suivis de cultivars plantains et du cultivar doux/Gros Michel qui vient en dernière position. Ceci a été rapporté dans les études menées par Efanden et al. (2005) et confirmées par

\section{CONCLUSION ET SUGGESTIONS}

L'objectif de notre travail étaient d'identifier le meilleur cultivar de bananier qui répond mieux à la méthode PIF en vue de mettre en place une technique de multiplication des plants au sein de châssis de propagation pouvant permettre aux agriculteurs d'accroître leur production. Les résultats obtenus de cette étude ont montré que le nombre total de plantules sevrées par explants le plus élevé a été observé chez les deux cultivars de bananier à cuire respectivement avec 36 et 34 plantules suivi de plantain Bubi avec 31 plantules. Tandis que le nombre total de plantules le plus faible a été observé chez le Dessert Gros les cultivars (PPDS = 2,017). Quant au nombre total de plantules sevrées par explants, le nombre le plus élevé a été observé chez les bananiers à cuire Saba et Cardaba (cv. ABB) respectivement avec 35 et 34 plantules, suivi du plantain Bubi (cv. AAB) avec 31 plantules. Tandis que, le nombre total de plantules le plus faible a été observé chez le Dessert Gros Michel (cv. AAA) avec 23 plantules. En outre, l'analyse statistique au seuil de $5 \%$ de probabilité indique des différences significatives entre les six cultivars (PPDS = 3,684). II en est de même pour le paramètre nombre total de plantules obtenues par cultivar (PPDS = $69,473)$. Les cultivars Gros Michel et Yangambi km5 ont présenté le nombre de plantules inférieur par rapport aux autres cultivars mis en essai. Ceci pourrait être dû par la présence (dominance) du triple génome Acuminata (AAA).

Lepoint et al. (2011). Ce comportement est différent de celui observé in vitro avec les cultivars du groupe génomique AAA pour lesquels le taux de multiplication est généralement plus élevé que pour les cultivars possédant un gène $B$ (Kwa, 1998a). Il est important de signaler que, les données de prolifération des plantules présentées sont presque similaires à ceux issus des études menées par Lepoint et al. (2011) dont le cultivar Saba a présenté une production élevée de 36 plantules comparativement aux 33 plantules avec PITA21 (AAB plantain hybride) obtenues par Lepoint et al. (2011). Ces résultats sont en accord avec ceux obtenus par Kwa $(2003,2009)$ qui a également observé que le nombre total des plantules par explant varie en fonction de cultivar. A partir d'un rejet, l'on est capable d'obtenir 10 à 100 plants de bananier plantain en fonction du cultivar et de l'expérience du manipulateur (Phaka and Bakelana, 1998). L'utilisation d'un cultivar approprié dans la technique de PIF permet d'obtenir plusieurs plants à la fois sains, moyennement uniformes et en seulement trois ou quatre mois (Mayeki et al., 2010; Ongagna et al., 2016).

Michel avec 23 plantules. A cet effet, tous ces cultivars peuvent être retenus et utilisés dans la technique de macro-propagation en vue d'augmenter la production de matériel de plantation de cette culture en un temps record mais en mettant un accent particulier sur le plantain Bubi dans la Ville de Kinshasa et ses environs. La technique des PIF devrait toutefois pouvoir être encore affinée, notamment par la mise au point d'un meilleur contrôle du nombre de cycles de proliférations in vivo. Des travaux ultérieurs devraient permettre de répondre à terme à ces différentes préoccupations. 


\section{REMERCIEMENTS}

Les auteurs adressent leur gratitude au "Bioversity International" pour la bourse recherche accordée à Bangata Bitha nyi Mbunzu.

\section{REFERENCES BIBLIOGRAPHIQUES}

Arias P, Dankers C, Liu P, Pilkauskas P, 2003. L'économie mondiale de la banane 1985- 2002. Food and Agriculture Organization of the United Nations (FAO), 102p.

Bakelana BK et Muyunga T, 1998. La production de bananes et de bananes plantain en République Démocratique du Congo. In Les productions bananières : un enjeu économique majeur pour la sécurité alimentaire, Pioq, C., Fouré, E. and Frison, E.A. (eds). Bananas and Food Security : International symposium. INIBAP, 103-115.

Bonte E, Verdonck R, Gregoire L, 1995. La multiplication rapide du bananier et du plantain au Cameroun. Tropicultura, 13(3) : 109-116.

Dhed'adjailo B, Mongo Manga A, Swennen R, 2011. La culture des bananiers et bananiers plantains en R.D. Congo. Support didactique, Edition Saint Paul Afrique, Kinshasa, 82p.

Efanden C, Kwa M, Temple L, Lescot T, 2005. Partenariat en sélection participative sur bananiers plantains : l'expérience du Centre africain de recherches sur bananiers et plantains au Cameroun. Partenaires pour construire des projets de sélection participative, Mars 2005, Cotonou, Benin, pp.153-173.

Kasyoka MR, Mwangi M, Kori N, Gitonga N, Muasya R, 2010. Evaluating the macropropagation efficiency of banana varieties preferred by farmers in Eastern and Central Kenya. Research Application Summary : Second RUFORUM Biennial Meeting 20 - 24 September 2010, Entebbe, Uganda, 499503.

Kwa M, 1998a. Étude des techniques de multiplication du matériel végétal in vivo, in : Rapport Technique 1996-1997, Doc. CRBP Njombé, 96-100.

Kwa M, 1998b. Le matériel de plantation : base d'une dynamique des productions bananières durables, in: Picq, C., Fouré, E., Frison, E.A. (Eds.), Les productions bananières : un enjeu économique majeur pour la sécurité alimentaire, INIBAP, Symp. Int., Douala, Cameroun.

Kwa M, 2003. Activation de bourgeons latents et utilisation de fragments de tige du bananier pour la propagation en masse de plants en conditions horticoles in vivo. Fruits, 58(6): 315-328.
Kwa M, 2007. Technique horticole de multiplication de masse de plantations, une innovation au service du développement, centre de recherches sur le bananier plantain (CARBAP), Nyombe, $8 \mathrm{p}$.

Kwa M, 2009. La culture et la multiplication des plants de bananier (Musa sp.), Connaissances et techniques de base, CARBAP, RD Congo, 13p.

Lepoint P, Iradukunda F and Blomme G, 2011. Macropropagation of Musa spp. In Burundi : A Preliminary Study. In: Guy Blomme, Bernard Vanlauwe and Piet van Asten (Ed.), Banana Systems in the Humid Highlands of Sub-Saharan Africa, Enhancing Resilience and Productivity, International conference organized by the Consortium for Improving Agriculture-based Livelihoods in Central Africa (CIALCA), Kigali, Rwanda, from 24 to 27 October 2011. pp. 58-65.

Manzur-Macia D, 2001. Propagation en masse in situ de l'hybride de bananier plantain FHIA- 20 par emploi de benzylaminopurine. Infomusa, 10(1): 3 $-4$.

Mayeki JP, Ndong Biyo'o M, Ngnigone Ella C, Molouba F, Demikoyo D, Mibemu S, Effa B, 2010. Influence de la composition des substrats sur le sevrage des vivoplants de plantains (Musa sp), Laboratoire de Biotechnologies Végétales, IRAFCENAREST in Sciences Sud, 3: 1-16.

Meutchieye F, 2009. Fiche technique de multiplication des bananiers par méthode de PIF, plants issus de fragments de tiges, manuel de formation pour la communauté rurale, $6 p$.

Mobambo KN, Staver C, Hauser S, Dheda B and Vangu G, 2010. An innovation capacity analysis to identify strategies for improving plantain and banana productivity and value addition in the Democratic Republic of Congo. Acta Horticulture (ISHS), 879: 821-827.

Ngo-Samnick EL, 2011. Production Améliorée du Bananier Plantain. Pro-Agro (Engineers Without Borders, Cameroon (ISF Cameroun)/Technical Centre for Agricultural and Rural Co-operation (CTA), Wageningen, The Netherlands.

Njeri Njau, Mwangi M, Kahuthia-Gathu R, Muasya R \& Mbaka J, 2012. Effectiveness of macropropagation technology in production of 
disease free banana seedlings in central and eastern Kenya, Research Application Summary : Third RUFORUM Biennial Meeting 24 - 28 September 2012, Entebbe, Uganda, 1327-1330.

Nkendah R and Akeyeampong E, 2003. Données socioéconomiques sur la filière plantain en Afrique Centrale et de l'Ouest. Infomusa, 12(1): 8 $-13$.

Ongagna A, Mialoundama F, Bakouetila MGF, 2016. Étude de la production des plants de bananiers et plantains (Musa spp.) par la technique des PIF au Congo: Effets des substrats sur la croissance et le développement des plants en pépinière, International Journal of Neglected and Underutilized Species (IJNUS) 2: 42-56.

Phaka V and Bakelana B, 1998. Multiplication de rejets de bananiers par fragmentation de la souche. In: Akyeampong, E. (ed.) Musa Network for West and Central Africa, Report of the Second Steering Committee Meeting, Douala, Cameroon. International Network for the Improvement of Banana and Plantain (INIBAP), Montpellier, France, pp. 51-52.

Sadom L, Tomekpé K, Folliot M, Côte FX, 2010. Comparaison de l'efficacité de deux méthodes de multiplication rapide de plants de bananier à partir de l'étude des caractéristiques agronomiques d'un hybride de bananier plantain (Musa sp.). Fruits, 65(1): 3-9.

Temple L, Kwa M, Fogain R, Mouliom Pefoura A, Bikoi A, 2002. Enjeux du plantain au Cameroun et amélioration des systèmes de production par une recherche-action participative In Acorbat. Memorias XV réunion. Realizada en Cartagena de Indias, Colombia. 27 de octobre al 02 novembre 2002; Sesión cartel transferencia de tecnología, CARBAP, S/C CIRAD BP 2572 Yaoundé. pp.593-601.

Youmbi $E$ et Ngaha D, 2004. Expression in vitro des capacités organogènes des bourgeons axillaires chez le bananier plantain (Musa sp.), Fruits, 59(04): 241-248. 